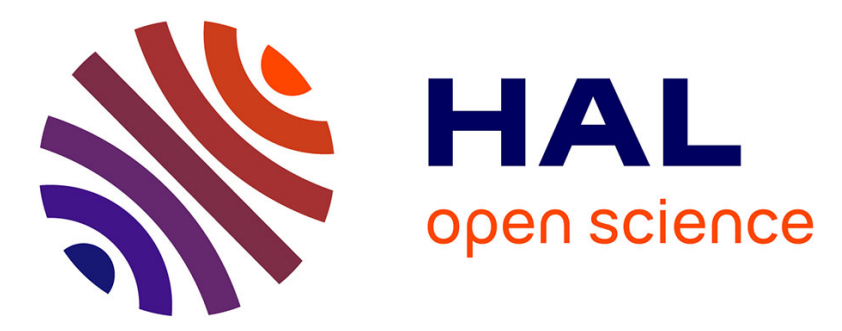

\title{
Ab initio investigation of perovskite and post-perovskite CaPtO3
}

\author{
Samir F. Matar, Gérard Demazeau, Alain Largeteau
}

\section{To cite this version:}

Samir F. Matar, Gérard Demazeau, Alain Largeteau. Ab initio investigation of perovskite and postperovskite CaPtO3. Chemical Physics, 2008, 352 (1-3), pp.92-96. 10.1016/j.chemphys.2008.06.005 . hal-00324657

\section{HAL Id: hal-00324657 \\ https://hal.science/hal-00324657}

Submitted on 25 Sep 2008

HAL is a multi-disciplinary open access archive for the deposit and dissemination of scientific research documents, whether they are published or not. The documents may come from teaching and research institutions in France or abroad, or from public or private research centers.
L'archive ouverte pluridisciplinaire HAL, est destinée au dépôt et à la diffusion de documents scientifiques de niveau recherche, publiés ou non, émanant des établissements d'enseignement et de recherche français ou étrangers, des laboratoires publics ou privés. 


\title{
$\mathrm{Ab}$ initio investigation of perovskite and post-perovskite $\mathrm{CaPtO}_{3}$
}

Samir F. Matar, Gérard Demazeau, Alain Largeteau

\begin{abstract}
For the recently synthesized post-perovskite (PPV) $\mathrm{CaPtO}_{3}$ we investigate the electronic band structure properties in connection with hypothetical perovskite $\mathrm{PV}-\mathrm{CaPtO}_{3}$ within density functional theory using pseudopotential and all electron methods. Both systems are found insulating in agreement with a tetravalent $\mathrm{Pt}(\mathrm{IV})$ low spin $\mathrm{d}^{6}$ state. The equilibrium values of the volume and energy point to a stabilization of the PPV versus PV oxide with a smaller volume in agreement with other homologue systems within Earth lower mantle such as $\mathrm{PPV}-\mathrm{MgSiO}_{3}$. This is also addressed from the analysis of the chemical bonding focusing on the change of the $\mathrm{Pt}-\mathrm{O}$ bonding upon going from PV and PPV systems whereby the covalent Pt-O bonds are differentiated and reinforced within PPV system with respect to $\mathrm{PV}$.
\end{abstract}

\section{Introduction}

The Earth lower mantle is mainly composed of $\mathrm{MgSiO}_{3}$ with the perovskite structure [1]. On the basis of such composition and structure many unusual properties of this deep part of the Earth (D" layer) were impossible to explain. In 2004, using both ab initio simulations and high pressure- high temperature experiments Oganov et al. [2] and Murakami et al. [3] underlined that the $\mathrm{D}^{\prime \prime}$ layer $\mathrm{MgSiO}_{3}$ will be characterized by a structural transformation from perovskite-type to the $\mathrm{CalrO}_{3}-$ type $(P \sim 120 \sim \mathrm{GPa}, T \sim 3000 \mathrm{~K})$. Since this new structure is denser than the perovskite $\mathrm{ABO}_{3}$ one (density discontinuity $\sim 1.4 \%$ ), it was called "post-perovskite structure" (PPV). The base-centered orthorhombic $\left(\mathrm{Cmcm}, \mathrm{N}^{\circ} 63\right) \mathrm{CalrO}_{3} \mathrm{PPV}$ structure is shown in Fig. 1a. It is characterized by corner as well as edge sharing $\mathrm{BO}_{6}$ octahedra in a closely two dimensional arrangement; the biggest $\mathrm{Ca}$ cation adopting the eight fold-coordination. This is contrary to perovskite $\mathrm{PV}$ structure $\left(\mathrm{Pbnm}, N^{\circ} 62\right)$ which is solely characterized by corner sharing octahedra (Fig. 1b). In both structures the $\mathrm{BO}_{6}$ octahedra are characterized by 2 different oxygen atoms, which are 2-fold (O1) and 4-fold (O2). This $\mathrm{CalrO}_{3} \mathrm{PPV}$ structure has been observed or proposed from calculations for different materials and different anions, such as $\mathrm{MgGeO}_{3}$ [4], [5] and [6], $\mathrm{NaMgF}_{3}$ [7], $\mathrm{UScS}_{3}$ [8], $\mathrm{ThMnSe}_{3}$ [9], $\mathrm{LaYbSe}_{3}[10], \mathrm{KTml}_{3}$ [11], $\mathrm{CaRuO}_{3}$ [12] and $\mathrm{MnGeO}_{3}$ [13] The stabilization of the post-perovskite structure for $\mathrm{CaPtO}_{3}[14]$ has underlined two different factors to play an

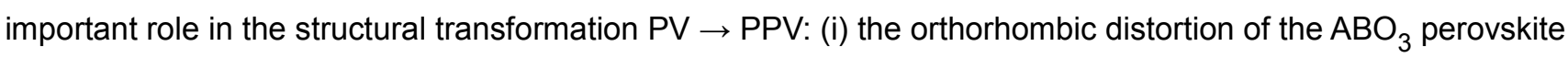
structure, in particular the tilting of the $\left(\mathrm{BO}_{6}\right)$ octahedra. Such a factor has been underlined recently through the re-investigation of the structural deformation of the $\mathrm{MgSiO}_{3}$ perovskite versus pressure [15], (ii) the covalence of the $\mathrm{B}-\mathrm{X}$ bond and its change, where $\mathrm{X}$ is an anion replacing $\mathrm{O}$. In fact all the PPV compounds prepared at 
ambient pressure contain $X$ anions with relatively low Pauling's electronegativity, $X_{\mathrm{p}}$ such as $\mathrm{S}$, Se and I or B cations with relatively high $X_{\mathrm{p}}$, such as Ir. The preparation of new $\mathrm{ABO}_{3}$ compositions with the PPV structure, in particular with B cations characterized by unpaired electrons, appears a challenge for the solid state chemist, the layered structure and shared-edges octahedral sites for B cations being able to induce specific anisotropic magnetic properties-due in particular to strong electron-electron correlations. Such post-perovskite structural type is an illustration of how a problem issued from Geosciences (the unusual behaviour of the D" layer) can bring new highlights in Materials Chemistry (a new structural type for $\mathrm{ABX}_{3}$ materials). For instance, the sodium substituted $\mathrm{CaIrO}_{3}\left(\mathrm{Ca}_{1-x} \mathrm{Na}_{x} \mathrm{IrO}_{3}\right)$, with a quasi two-dimensional lattice is characterized by a metal-insulator transition and a simultaneous change of the magnetic ground state from an antiferromagnetic to a paramagnetic state [16].

This work which calls for modeling theoretical tools within the well established framework of density functional theory (DFT) [17] and [18], is devoted to establish a comparative electronic structure and chemical bonding study between the actual PPV-CaPtO 3 and hypothetic $\mathrm{PV}-\mathrm{CaPtO}_{3}$, for the purpose of assessing origins for the stability of PPV systems such as $\mathrm{MgSiO}_{3}$.

\section{Theoretical framework}

\subsection{Computational methods}

Two computational methods were used within the DFT. A pseudo potential approach within the VASP code [19] and [20] was firstly used to optimize the structure for hypothetic $\mathrm{PV}-\mathrm{CaPtO}{ }_{3}$ and then confront its volume and stability relative to $\mathrm{PPV}-\mathrm{CaPtO}_{3}$ through energy-volume curves. For such a procedure pseudo-potentials for the different chemical species, built within the local density approximation (LDA) [21], were used. The calculations were converged at an energy cut-off of $395.7 \mathrm{eV}$ for $\mathrm{PV}-\mathrm{CaPtO}_{3}$ and $494.62 \mathrm{eV}$ for $\mathrm{PPV}-\mathrm{CaPtO}_{3}$ for the planewave basis set. The $k$-point integration was carried out with a starting mesh of $4 \times 4 \times 4$ up to $8 \times 8 \times 8$ for best convergence and relaxation to zero strains. The Brillouin-zone integrals were approximated using a special $k$ point sampling [22].

For a full description of the electronic structures and of chemical bonding, the scalar relativistic all-electrons augmented spherical wave (ASW) [23] and [24] method was used. The calculations are likewise based on the DFT with use of an LDA scheme [25] for an account of exchange-correlation effects. In the ASW method, the wave function is expanded in atom-centered augmented spherical waves, which are Hankel functions and numerical solutions of Schrödinger's equation, respectively, outside and inside the so-called augmentation spheres. In order to optimize the basis set, additional augmented spherical waves were placed at carefully selected interstitial sites (IS). The choice of these sites as well as the augmentation radii were automatically determined using the sphere-geometry optimization algorithm [26]. Self-consistency was achieved by a highly efficient algorithm for convergence acceleration [27]. The Brillouin zone integrations were performed using the linear tetrahedron method with up to 1469 k-points within the irreducible wedge [22] and [24]. In the minimal ASW basis set, we chose the outermost shells to represent the valence states using partial waves up to $I_{\max }+1=4$ for $\mathrm{Pt}, I_{\max }+1=3$ for $\mathrm{Ca}$ and $I_{\max }+1=2$ for $\mathrm{O}$. Low energy lying $\mathrm{O}(2 \mathrm{~s})$ states were considered as 
core states in the all electrons calculations. Further, considering empty $\mathrm{O}(3 \mathrm{~s})$ states in the valence set allows for a more complete basis set. The completeness of the valence basis set was checked for charge convergence. The self-consistent field calculations are run to a convergence of $\Delta Q=10^{-8}$ for the charge density and the accuracy of the method is in the range of about $10^{-8}$ Ryd regarding energy differences.

\subsection{Assessment of the chemical bonding}

To extract detailed information about the nature of the interactions between the atomic constituents from electronic structure calculations, the crystal orbital overlap population (COOP) [28] or the crystal orbital Hamiltonian population (COHP) [31] may be employed. While both the COOP and COHP approaches provide a qualitative description of the bonding, nonbonding, and antibonding interactions between two atoms, the COOP description in some cases exaggerates the magnitude of antibonding states. A slight refinement was recently proposed in form of the so-called covalent bond energy ECOV criterion, which combines the COHP and COOP to calculate quantities independent of the particular choice of the potential zero [32]. In the present work this covalent bond energy criterion implemented within the ASW method, was used for the chemical bonding analysis. In the plots, negative, positive and zero unitless magnitudes of ECOV are indicative of bonding, antibonding, and nonbonding interactions respectively.

\section{Geometry optimization results}

Based on the structural determination data of $\mathrm{PV}-\mathrm{CalrO}_{3}$ [33], a full geometry relaxation was carried out for a hypothetical $\mathrm{PV}-\mathrm{CaPtO}_{3}$ in order to confront its equilibrium properties with those of $\mathrm{PPV}-\mathrm{CaPtO}{ }_{3}$. For the latter, crystal parameters determined for the system synthesized under high pressure conditions [14] were used for the calculations. The results of geometry optimization reproduce the internal parameters of the literature for Ca, and

$\mathrm{O}$ positions, with $\mathrm{Pt}$ kept at the origin $(0,0,0)$ in both systems. The equilibrium cell volume $\mathrm{V}_{0}$ for $\mathrm{PPV}-\mathrm{CaPtO}{ }_{3}$ is 212.14 $\AA^{3}$, smaller than the experimental value of $228 \AA^{3}$ [14]. Besides the approximations of the computational method, this is a common feature due to the use of the LDA which tends to overestimate the binding - hence leading to smaller lattice spacings - in as far as it is built within the electron gas scheme [21]. We note here that testing other DFT functionals such as a generalized gradient one (GGA) [29] or LDA + U [30] which boosts correlation, did not lead to significant improvements of the calculated volume magnitude with respect to experiment. It is interesting to note that the volume of the geometry optimized $\mathrm{PV}-\mathrm{CaPtO}{ }_{3}, V_{0}=214.75 \AA^{3}$ is slightly larger than that of PPV phase obtained from calculations carried out in the same computational conditions. This points to a denser PPV system, in agreement with experimental observations for such PPV systems as discussed in the introduction. As for the energetics, the PPV-CaPtO 3 is found more stable the PV$\mathrm{CaPtO}_{3}$ by $\Delta E=-0.191 \mathrm{eV}$. This energy difference corresponds to $\Delta E=18.4 \mathrm{~kJ} \mathrm{~mol}^{-1}$. This is intermediate between the calculated value $\left(\Delta E=11.8 \mathrm{~kJ} \mathrm{~mol}^{-1}\right)$ and the reported calorimetric value $\left(\Delta E \sim 32 \mathrm{~kJ} \mathrm{~mol}^{-1}\right)$ for $\mathrm{CalrO}_{3}$ with the same trend of a larger stability for the PPV system [33]. We explicit these features from the change of the total energy with unit cell volume around equilibrium as shown in Fig. $2 a$ and $b$ for PPV and PV $\mathrm{CaPtO}_{3}$ respectively. Note that due to $\mathrm{C}$ centering (with one additional lattice point at the center of one of the cell faces) of PPV $(\mathrm{Cmcm})$ as opposed to the P primitive centering (lattice points on the cell corners) of PV (Pbnm), 
the calculations are done for half of the unit cell in the former and so are the volume and energy magnitudes. The quadratic fit of the $E(V)$ (energy versus volume) curves with a second order Birch equation of state [34] leads to calculated fitted parameters shown in the inserts. An interesting feature of a larger $B_{0}$ bulk module for PPV versus $\mathrm{PV}$ is concomitant with the smaller volume and denser atomic arrangement. We also note the larger magnitudes of our $\mathrm{B}_{0}$ values as with respect to those of $\mathrm{CalrO}_{3}$ [33], thus pointing to an overall harder $\mathrm{Pt}$ based system.

We now turn to develop the issues relevant to the relative stabilities of PPV versus $\mathrm{PV} \mathrm{CaPtO}_{3}$ in next section through a detailed analysis of the electronic band structure and of the chemical bonding.

\section{Band structure, DOS and chemical bonding}

Using the fitted lattice parameters from the geometry relaxation, we carried out all electrons calculations of the electronic band structure, the density of states (DOS) and of the chemical bonding using the ECOV criterion for $\mathrm{PPV}$ and $\mathrm{PV} \mathrm{CaPtO}{ }_{3}$. The band structures along the major Brillouin zone lines of base-centered orthorhombic (PPV) and simple orthorhombic (PV) Bravais lattices are shown in Fig. 3a and b. Two major features are observed:

(i) both systems are insulating, in agreement with a Pt ${ }^{\mathrm{IV}}$ state. The band gap for PPV (Fig. 3a) $E_{g} \sim 1.8 \mathrm{eV}$, is more than twice larger than in PV (Fig. 3b), $E_{\mathrm{g}} \sim 0.8 \mathrm{eV}$.

(ii) the valence band (VB) in PPV is broader by $\sim 1 \mathrm{eV}$ and characterized by a larger dispersion. This leads to suggest a larger covalence which could be related to the edge and corner sharing arrangement of the $\mathrm{PtO}_{6}$ in PPV while those octahedra are only corner sharing in PV (as well as in regular cubic perovskites!). It is also interesting to note the nearly flat band behavior at the top of the valence band as well as at the bottom of the conduction bands. The nature of these states is discussed in next paragraph with site projected DOS. The plots of the projected DOS (PDOS) for the constituent atom sites are presented in Figs. 4a and b. They account for the total number of atoms in the unit cell which contains, as discussed above, half as many atoms in the $C$ lattice centering (PPV) as in the P centering (PV), whence the relative DOS intensities along the $y$-axes. The DOS mirror the band structure features discussed above and add up details as to the states that mix together and as to their prevailance in the different energy windows. This is especially true for the relative band gap magnitudes and the presence of Pt $d$ states at both sides of the gap. The lower part of VB is responsible of the chemical bonding within the oxide system, i.e., mainly between itinerant $\mathrm{Pt}\left(\mathrm{d}-\mathrm{e}_{\mathrm{g}}\right)$ and $\mathrm{O}(2 \mathrm{p})$, assuming a nearly octahedral surrounding of Pt. This is observed from the similar skylines of the PDOS below $-4 \mathrm{eV}$. This feature is more pronounced in $\mathrm{PPV}-\mathrm{CaPtO}_{3}$ for 4-fold $\mathrm{O} 2$ with respect to 2-fold $\mathrm{O} 1$ while no differentiation occurs for PV$\mathrm{CaPtO}_{3}$

The DOS results can be further assessed in a chemical bonding qualitative analysis within the ECOV criterion introduced above. The plots are shown in Fig. 5a and b; they provide a picture of a VB that is half bonding up till $-4 \mathrm{eV}$ and half antibonding between $-4 \mathrm{eV}$ and the top of the VB. This is due to the large filling of $\mathrm{Pt} d$ states whereby extra electrons are arranged into antibonding states. This is opposite to $\mathrm{MgSiO}_{3}$ which is studied for comparison in the PPV phase in next paragraph. While the relative $\mathrm{Pt}-\mathrm{O} 1$ and $\mathrm{Pt}-\mathrm{O} 2 \mathrm{ECOV}$ are the same in the 
perovskite system, there is clearly a differentiation of the bonding within the post-perovskite oxide. This can arise from two possible origins pertaining to the change in distances or to the arrangement of the octahedra as shown in Fig. 1. Concerning the distances, let's note here the relevant $\mathrm{Pt}-\mathrm{O} 1$ and $\mathrm{Pt}-\mathrm{O} 2$ distances (in atomic units): 3.83/3.89 for PV and 3.74/3.80 for PPV, i.e., they differ by 0.06 a.u. in both varieties and cannot be responsible for the large changes in the bonding. Consequently one has to assign the changes in the bonding to an increased covalency induced by the corner as well as edge sharing octahedra through 4-fold oxygen sites, $\mathrm{O} 2$ while the Pt-O1 bonds are weakened.

We also stress the results by the study of PPV $-\mathrm{MgSiO}_{3}$ for the PDOS and ECOV. The results are illustrated in Fig. $6 a$ and $b$ respectively. From Fig. $6 a$ the DOS features show a predominant oxygen like character at the top of the VB and a large dispersion of states at the bottom of the CB, indicative of $s, p$ like bands so that the energy gap is reduced with respect to PPV-CaPtO 3 homologue (cf. Fig. 3a) where Pt d states are dominant. The feature of a differentiated $\mathrm{B}-\mathrm{O}(\mathrm{B}=\mathrm{Si})$ bonding is observed in Fig. $6 \mathrm{~b}$ with the difference of a totally bonding character within the VB. But the same trend as in $\mathrm{PPV}-\mathrm{CaPtO}_{3}$ is observed, i.e., favoring one type of bond (Si-O2) over the other ( $\mathrm{Si}-\mathrm{O} 1)$ pertaining to a larger covalent character of the former.

\section{Conclusion}

Using theoretical modeling tools, we have assessed the stability of recently synthesized post-perovskite (PPV) $\mathrm{CaPtO}_{3}$ by an examination of its electronic and chemical bonding properties. This was established in a comparative discussion with perovskite (PV) arrangement for this system devised ab initio. The PPV system is found more stable than PV with a larger gap opening and a broader valence band which was argued upon by an increased covalency within PPV due to corner as well as edge sharing $\mathrm{BO}_{6}$ octahedra. This was equally argumented from a study of the change in the chemical bonding occurring within and between $\mathrm{PtO}_{6}$ octahedral arrangements in PPV. A generelization to PPV- $\mathrm{MgSiO}_{3}$ was also shown. From the results an emerging picture illustrating the mechanism of PV $\rightarrow$ PPV transformation can be sketched: The evolution of the tilting of the $\mathrm{BO}_{6}$ octahedra within PV structure increases with pressure until its complete destabilization and the subsequent formation of PPV. However the latter should only be stabilized if the B-O bond is sufficiently covalent for stabilizing layers of edge sharing $\mathrm{BO}_{6}$ octahedra. We believe that such studies should promote synthesis routes for new PPV systems. This is being currently investigated at our Institute.

\section{Acknowledgement}

Computational facilities were provided within the intensive numerical simulation facilities network M3PEC of the Université de Bordeaux (http://www.m3pec.u-bordeaux1.fr), partly financed by the Conseil Régional d'Aquitaine and the French ministry of research and technlogy.

\section{References}

[1] G. Fiquet, Z. Krist. 216 (2001), p. 248.

[2] A.R. Oganov and S. Ono, Nature 430 (2004), p. 445.

[3] M. Murakami, K. Hirose, K. Kawamura, N. Sata and Y. Ohishi, Science 304 (2004), p. 855. 
[4] K. Hirose, K. Kawamura, Y. Ohishi, S. Tateno and N. Sata, Am. Mineral. 90 (2005), p. 262.

[5] A. Kubo, B. Kieffer, G.Y. Shen, V.B. Prakapenka, R.J. Cava and T.S. Duffy, Geophys. Res. Lett. 33 (2006), p. L12.

[6] C.D. Martin, Y. Meng, V. Prakapenka and J.B. Parise, J. Appl. Crystallogr. 41 (2008), p. 38.

[7] C.D. Martin, W.A. Crichton, D.J. Weidner, D. Häusermann and H.K. Mao, Geophys. Res. Lett. 32 (2005), p. L04304.

[8] R. Julien, N. Rodier and V. Tien, Acta Crystallogr. B 34 (1978), p. 2612.

[9] I. Ijjali, K. Mitchell, F.Q. Huang and J.A. Ibers, J. Solid State Chem. 177 (2004), p. 257.

[10] K. Mitchell, R.C. Somers, F.Q. Huang and J.A. Ibers, J. Solid State Chem. 177 (2004), p. 709.

[11] G. Schilling, C. Kunert, T. Sschleid and G. Meyer, Z. Anorg. Allg. Chem. 618 (1992), p. 7.

[12] H. Kojitani, Y. Shirako and M. Akaogi, Phys. Earth Planetary Inter. 165 (2007), p. 127.

[13] S. Tateno, K. Hirose, N. Sata and Y. Ohishi, Phys. Chem. Miner. 32 (2006), p. 721.

[14] D.Y. Jung and A.R. Oganov, Phys. Chem. Miner. 32 (2005), p. 146.

[15] M. Sugahara, A. Yoshiasa, Y. Komatsu, T. Yamanaka, N. Bolfan-Casanova, A. Nakatsuka, S. Sasaki and M. Tanaka, Am. Mineral. 91 (2006), p. 533.

[16] K. Ohgushi, H. Gotou, T. Yagi, Y. Kiuchi, F. Sakai and Y. Ueda, Phys. Rev. B 74 (2004), p. 241104.

[17] P. Hohenberg and W. Kohn, Phys. Rev. B 136 (1964), p. 864.

[18] W. Kohn and L.J. Sham, Phys. Rev. A 140 (1965), p. 1133.

[19] G. Kresse and J. Furthmüller, Phys. Rev. B 54 (1996), p. 11169.

[20] G. Kresse and J. Joubert, Phys. Rev. B 59 (1999), p. 1758.

[21] J.P. Perdew and A. Zunger, Phys. Rev. B 23 (1981), p. 5048.

[22] P.E. Blöchl, Phys. Rev. B 50 (1994), p. 17953. ।

[23] A.R. Williams, J. Kübler and C.D. Gelatt, Phys. Rev. B 19 (1979), p. 6094.

[24] V. Eyert, The Augmented Spherical Wave Method - A Comprehensive Treatment, Lecture Notes in Physics vol. 719, Springer, Berlin/Heidelberg (2007).

[25] S.H. Vosko, L. Wilk and M. Nusair, Can. J. Phys. 58 (1980), p. 1200.

[26] V. Eyert and K.-H. Höck, Phys. Rev. B 57 (1998), p. 12727.

[27] V. Eyert, J. Comput. Phys. 124 (1996), p. 271.

[28] R. Hoffmann, Angew. Chem. Int. Ed. Engl. 26 (1987), p. 846.

[29] J.P. Perdew, S. Burke and M. Ernzerhof, Phys. Rev. Lett. 77 (1996), p. 3865. I

[30] V.I. Anisimov, J. Zaanen and O.K. Andersen, Phys. Rev. B 44 (1991), p. 943.

[31] R. Dronskowski and P.E. Blöchl, J. Phys. Chem. 97 (1993), p. 8617.

[32] G. Bester and M. Fähnle, J. Phys: Condens. Matter 13 (2001), p. 11541.

[33] S. Stølen and R. Tronnes, Phys. Earth Planet. In. 164 (2007), p. 50.

[34] F. Birch, J. Geophys. Res. 83 (1978), p. 1257. 
a

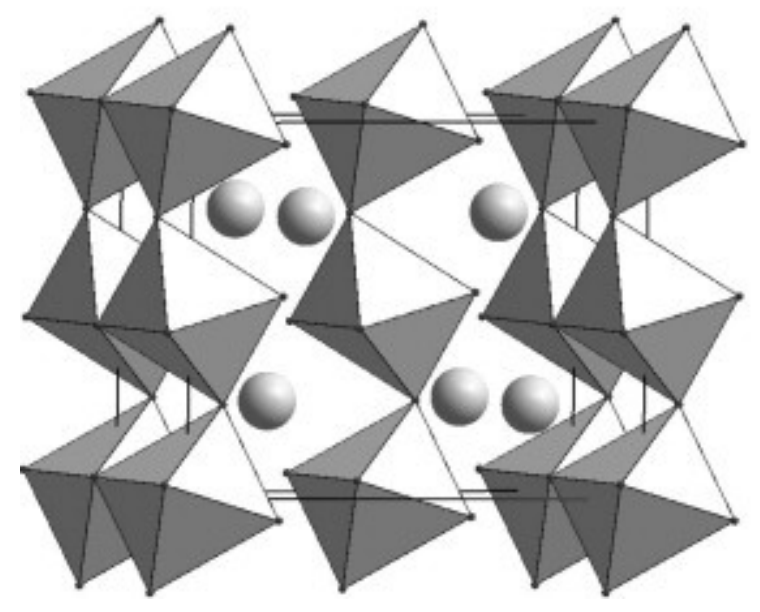

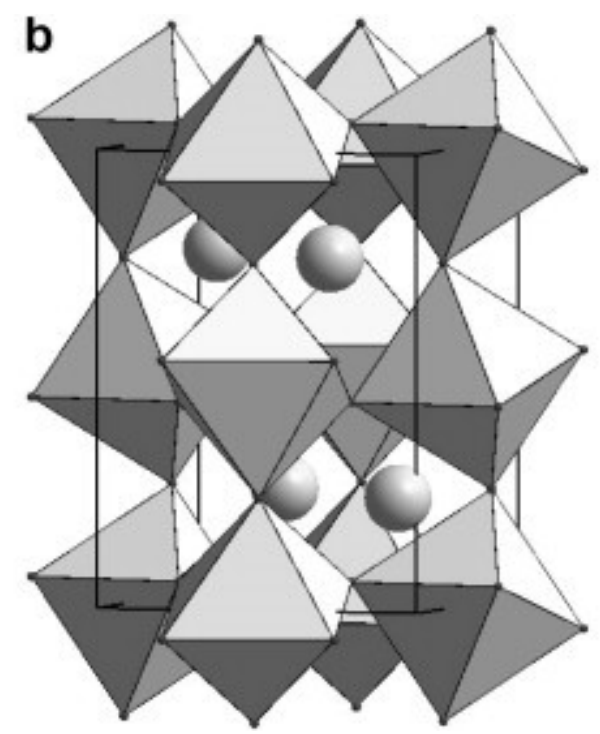

Fig. 1. Crystal structure of two varieties of $\mathrm{CaPtO}_{3}$ showing different arrangements of $\mathrm{PtO}_{6}$ octahedra in basecentered orthorhombic post-perovskite PPV (a) and in hypothetic orthorhombic perovskite PV (b). Grey spheres represent the $\mathrm{Ca}$ ions. See text for details. 

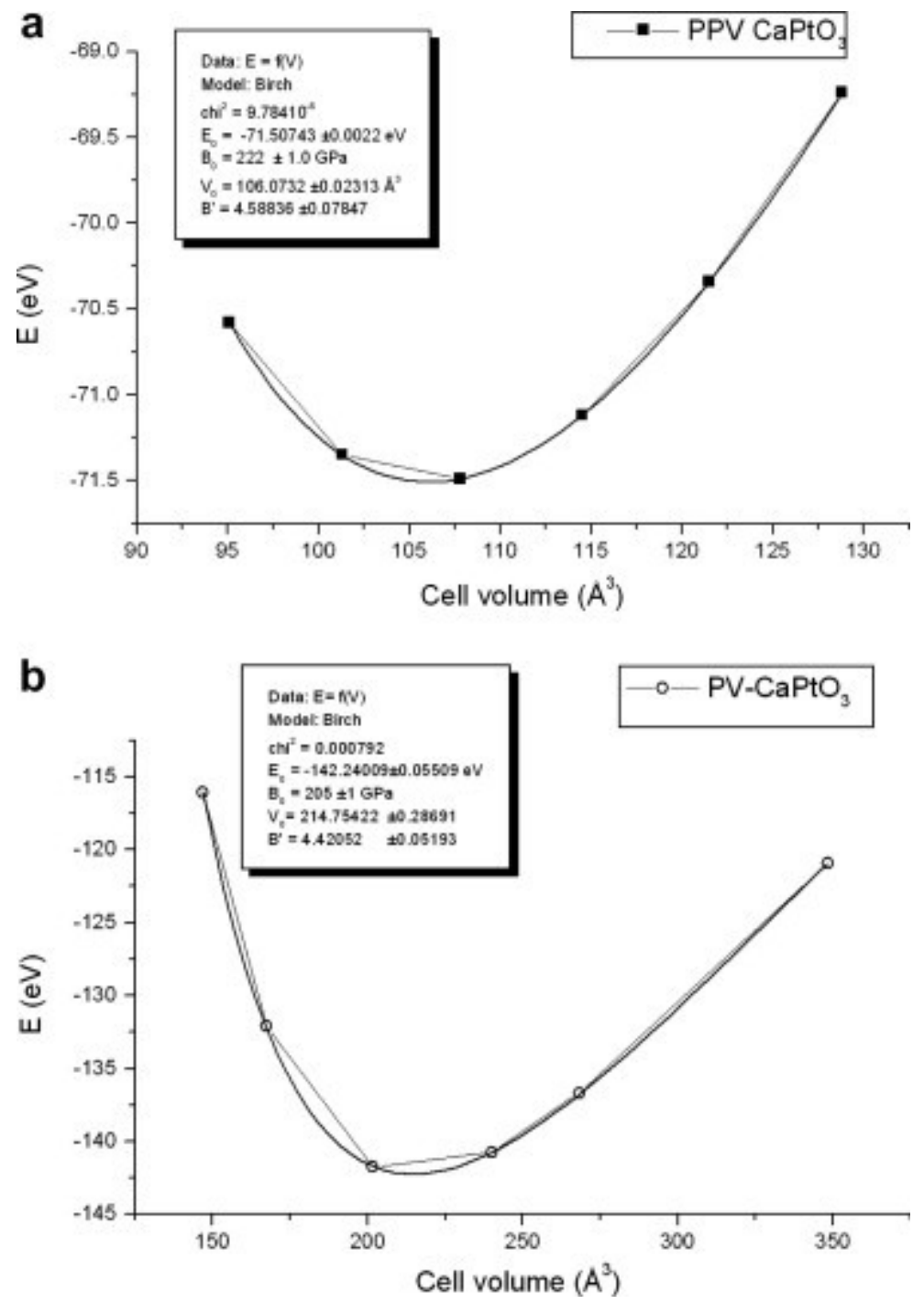

Fig. 2. Total energy versus cell volume curves for $\mathrm{PPV}-\mathrm{CaPtO}_{3}(\mathrm{a})$ and $\mathrm{PV}-\mathrm{CaPtO}_{3}$ (b). Fits were done using Birch second order equation of state. 

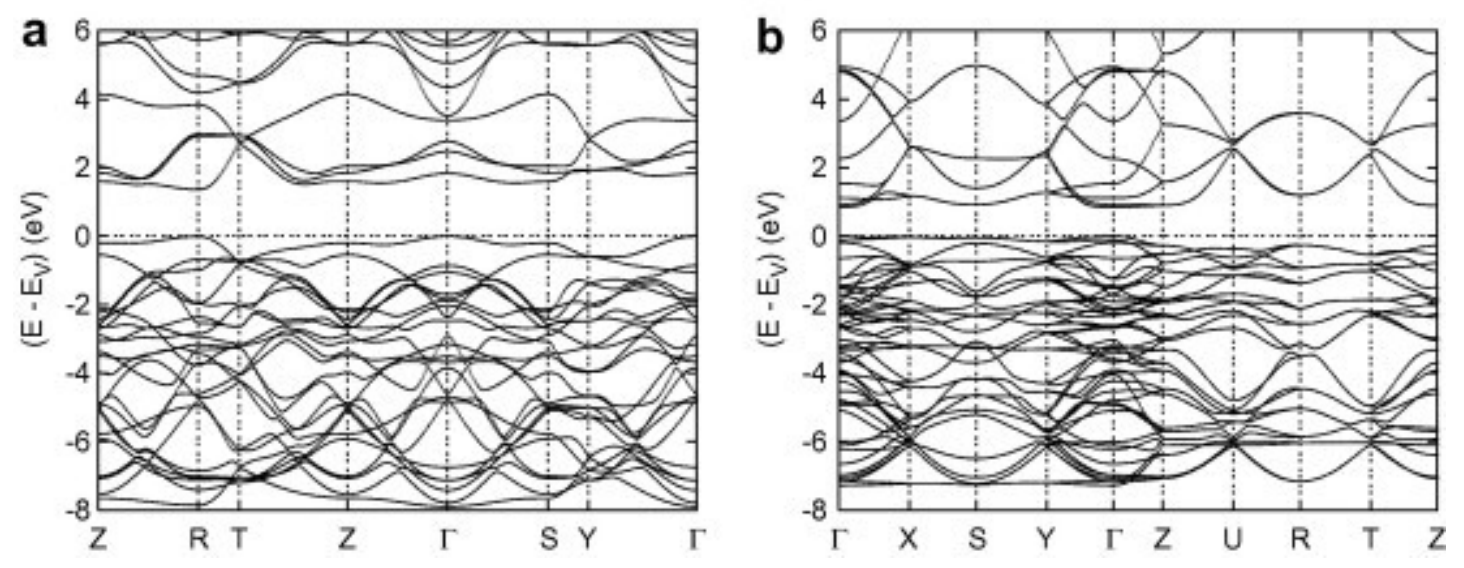

Fig. 3. Electron band structure of $\mathrm{CaPtO}_{3}:$ (a) PPV and (b) PV. Energy reference along $y$-axis is with respect to the top of the valence band $E_{\mathrm{V}}$. 

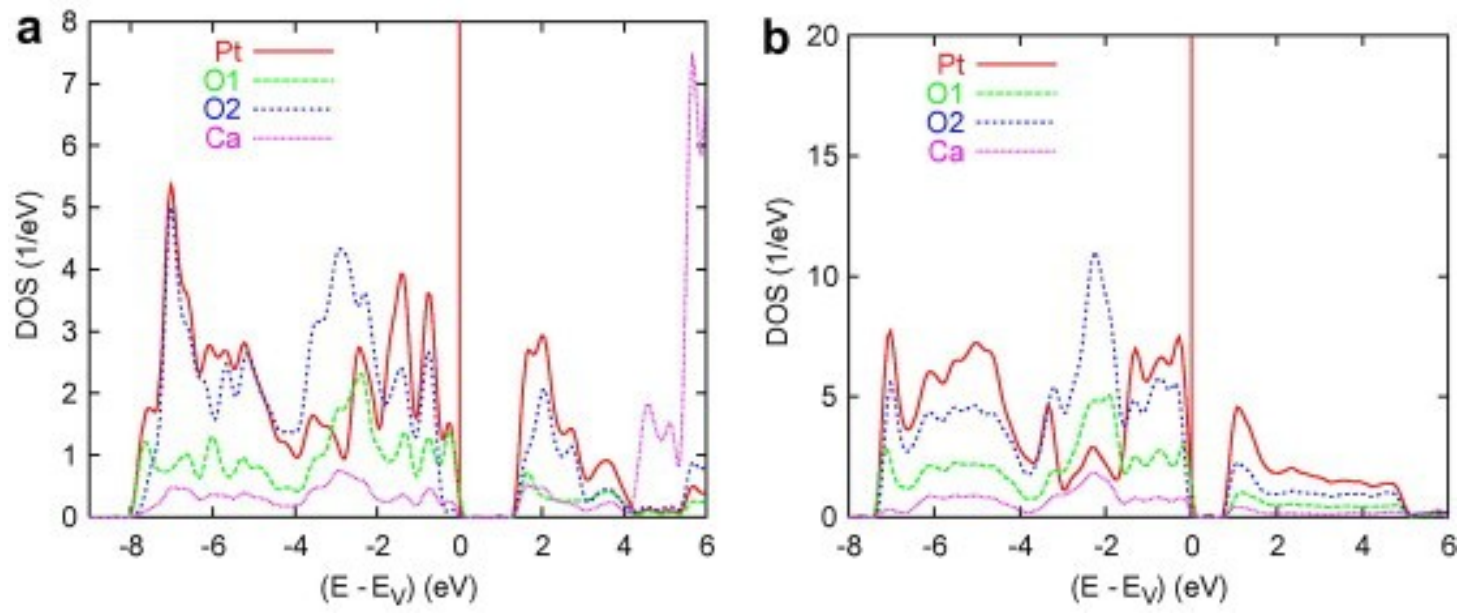

Fig. 4. (color online) Site projected DOS: $\mathrm{PPV}-\mathrm{CaPtO}_{3}$ (a) and PV-CaPtO 3 (b). 

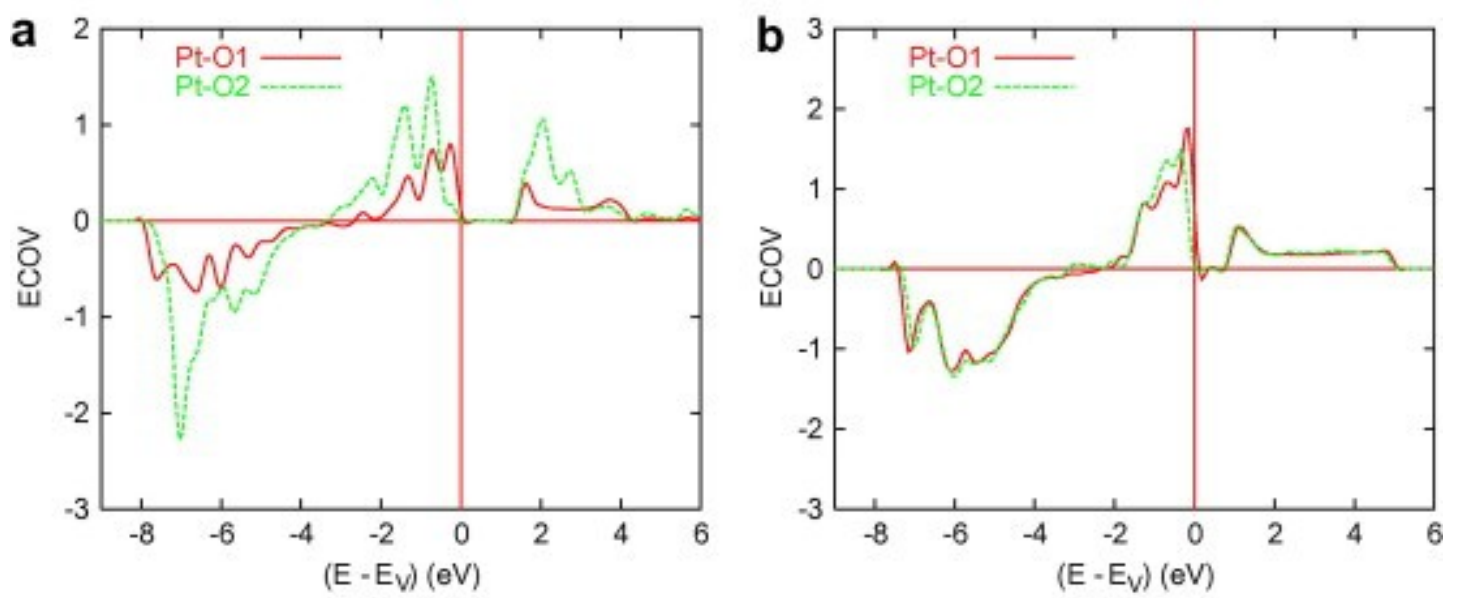

Fig. 5. (color online) Changes in the Pt-O chemical bond from ECOV criterion (unit-less) in (a) $\mathrm{PPV}-\mathrm{CaPtO}_{3}$ and (b) PV-CaPtO . $^{\text {. }}$ 

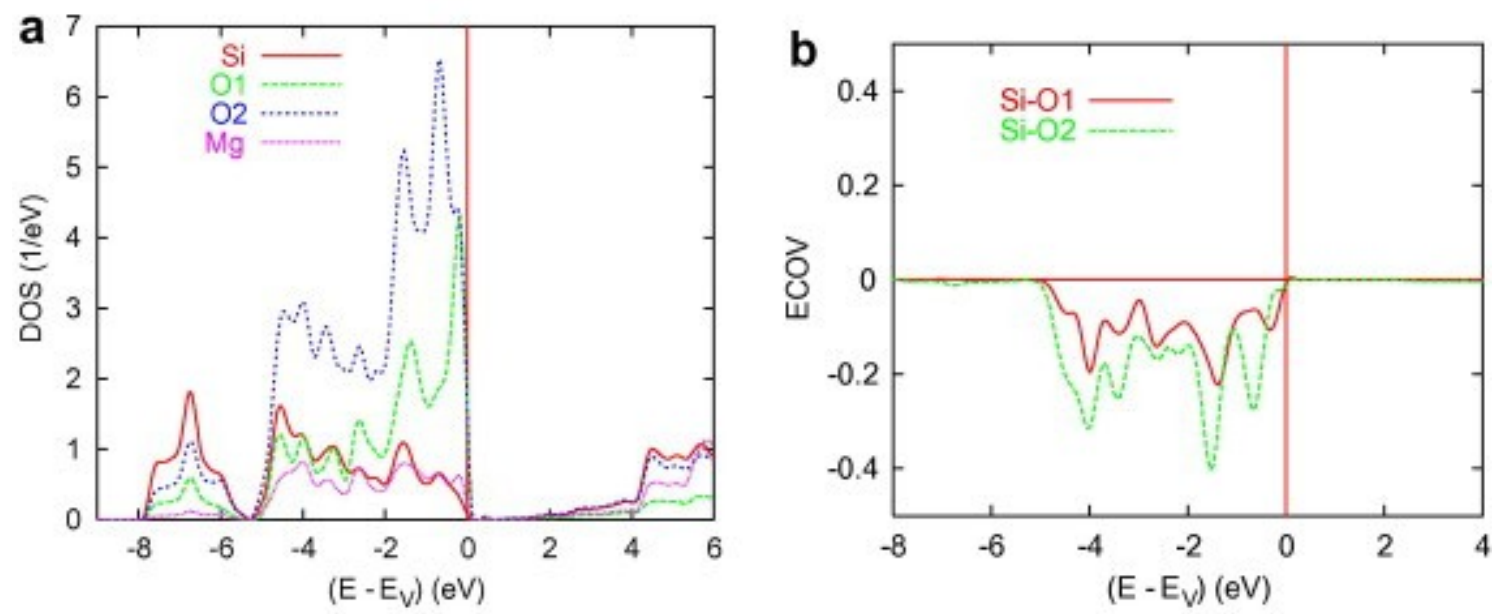

Fig. 6. (color online) Post-perovskite PPV-MgSiO $3:$ (a) site projected DOS and (b) Si-O chemical bonds. 